

\section{Wundmanagement - Wundversorgung in der täglichen Praxis}

Thilo Bode

Thomas Horn

Andrea Schüning

283 Abbildungen

Georg Thieme Verlag Stuttgart • New York 
Bibliografische Information der Deutschen Nationalbibliothek

Die Deutsche Nationalbibliothek verzeichnet diese Publikation in der Deutschen Nationalbibliografie; detaillierte bibliografische Daten sind im Internet über http://dnb.d-nb.de abrufbar.

Ihre Meinung ist uns wichtig! Bitte schreiben Sie uns unter:

www.thieme.de/service/feedback.html (c) 2022. Thieme. All rights reserved.

Georg Thieme Verlag KG

Rüdigerstraße 14, 70469 Stuttgart, Germany www.thieme.de

Dieses Buch entstand mit freundlicher Unterstützung der Helios Kliniken $\mathrm{GmbH}$.

Umschlaggestaltung: (C) Thieme

Bildnachweis Cover: (c) Thieme

Zeichnungen: Karin Baum, Paphos, Zypern;

Holger Vanselow, Stuttgart

Redaktion: Elisabeth Dominik, Allendorf/Lumda

Satz: Druckhaus Götz GmbH, Ludwigsburg

Druck: L.E.G.O. S.p.A., Vicenza

\section{DOI $10.1055 /$ b000 000446}

ISBN 978-3-13-243797-5
Wichtiger Hinweis: Wie jede Wissenschaft ist die Medizin ständigen Entwicklungen unterworfen. Forschung und klinische Erfahrung erweitern unsere Erkenntnisse, insbesondere was Behandlung und medikamentöse Therapie anbelangt. Soweit in diesem Werk eine Dosierung oder eine Applikation erwähnt wird, darf der Leser zwar darauf vertrauen, dass Autoren, Herausgeber und Verlag große Sorgfalt darauf verwandt haben, dass diese Angabe dem Wissensstand bei Fertigstellung des Werkes entspricht.

Für Angaben über Dosierungsanweisungen und Applikationsformen kann vom Verlag jedoch keine Gewähr übernommen werden. Jeder Benutzer ist angehalten, durch sorgfältige Prüfung der Beipackzettel der verwendeten Präparate und gegebenenfalls nach Konsultation eines Spezialisten festzustellen, ob die dort gegebene Empfehlung für Dosierungen oder die Beachtung von Kontraindikationen gegenüber der Angabe in diesem Buch abweicht. Eine solche Prüfung ist besonders wichtig bei selten verwendeten Präparaten oder solchen, die neu auf den Markt gebracht worden sind. Jede Dosierung oder Applikation erfolgt auf eigene Gefahr des Benutzers. Autoren und Verlag appellieren an jeden Benutzer, ihm etwa auffallende Ungenauigkeiten dem Verlag mitzuteilen.

Marken, geschäftliche Bezeichnungen oder Handelsnamen werden nicht in jedem Fall besonders kenntlich gemacht. Aus dem Fehlen eines solchen Hinweises kann nicht geschlossen werden, dass es sich um einen freien Handelsnamen handelt.

Das Werk, einschließlich aller seiner Teile, ist urheberrechtlich geschützt. Jede Verwendung außerhalb der engen Grenzen des Urheberrechtsgesetzes ist ohne Zustimmung des Verlages unzulässig und strafbar. Das gilt insbesondere für Vervielfältigungen, Übersetzungen, Mikroverfilmungen oder die Einspeicherung und Verarbeitung in elektronischen Systemen.

Wo datenschutzrechtlich erforderlich, wurden die Namen und weitere Daten von Personen redaktionell verändert (Tarnnamen). Dies ist grundsätzlich der Fall bei Patienten, ihren Angehörigen und Freunden, z. T. auch bei weiteren Personen, die z. B. in die Behandlung von Patienten eingebunden sind. Thieme nennt Autorinnen und Autoren konkrete Beispiele, wie sich die Gleichstellung von Frauen und Männern sprachlich darstellen lässt. Wo im Text (z.B. aus Gründen der Lesbarkeit) nur das generische Maskulinum verwendet wird, sind alle Geschlechter gleichermaßen gemeint.

Auch erhältlich als E-Book:

eISBN (PDF) 978-3-13-243798-2

eISBN (epub) 978-3-13-243799-9 


\section{Danksagung}

Wir danken allen Mitwirkenden, die diesem Werk zugearbeitet haben. Dies gilt insbesondere den Mitgliedern der AG Chronische Wunde für die umfangreiche Unterstützung bei der Erstellung dieses Buches. 


\section{Einleitung}

In den vergangenen 40 Jahren ist eine Vielzahl von Produkten zur Wundbehandlung auf den Markt gekommen. Schlagworte wie „feuchte Wundbehandlung“ oder „Wundmanagement“ haben teilweise eher zur Verwirrung als zur Klärung beigetragen. Auf den ersten Blick scheinen viele neue Produkte teurer als „altbewährte“ Vorgehensweisen. Jedoch bieten sie neben einer oft schnelleren Befundbesserung bzw. Abheilung von Wunden auch therapeutische Möglichkeiten bei bisher „infausten Wundzuständen“. Die Fülle der Produkte erfordert ein sehr differenziertes Wissen, um ihren adäquaten und dennoch kostengünstigen Einsatz zu ermöglichen.

Die Helios-Geschäftsführung beschloss daher die Einrichtung einer Arbeitsgruppe „Spezielle Wundversorgung“. Zielsetzung war es, die auf dem Markt angebotene Produktpalette zu sichten und Anwendungsempfehlungen zu erarbeiten, die die klinischen Belange der verschiedenen medizinischen Fachrichtungen berücksichtigen sollten. Letztlich sollte damit eine möglichst einheitliche Vorgehensweise erzielt werden. Dies beinhaltet auch die Abschaffung „alter Zöpfe“ sowie die Information über neue, z.T. wesentlich effektivere Behandlungsverfahren.

Von den auf dem Markt erhältlichen Präparaten wurde durch die Arbeitsgruppe anhand von Überprüfungskriterien (Produkteigenschaften, Handhabung, Verpackungsmaterial, Kosten etc.) die für die Helios-Kliniken bestgeeignete Auswahl getroffen. Die Sortimentsanpassung erfolgt regelhaft alle drei Jahre. Die hier vorliegende Wundfibel „Wundmanagement - Wundversorgung in der täglichen Praxis“ enthält neben Informationen über die einzelnen ausgewählten Produkte auch Therapievorschläge zur Behandlung verschiedener Wunden und Wundstadien.

Die für den Inhalt der Wundfibel verantwortliche AG wurde inzwischen aufgrund eines erweiterten Aufgabenspektrums in AG „Chronische Wunde“ umbenannt. Die jetzt vorliegende neue Version der „HELIOS Wundfibel“ wurde in Anlehnung an die Krefelder Wundfibel völlig umstrukturiert und neu erstellt. Sie wurde von der Helios-Geschäftsführung als verbindlich genehmigt.

Die Wundfibel ist als Beitrag zur Qualitätssicherung zu werten und bedarf einer kontinuierlichen Bearbeitung und Aktualisierung.

Neben einem allgemeinen Teil zur Wundversorgung folgt in einem weiteren Teil eine ausführliche Auflistung von Präparategruppen zur Wundbehandlung. Hierbei ist das jeweils bei Helios bevorratete Produkt in seinen gelisteten Größen aufgeführt. Darüber hinaus werden Alternativprodukte anderer Hersteller ohne irgendeinen Anspruch auf Vollständigkeit und Richtigkeit der Bezeichnung nur namentlich aufgeführt, da dies ansonsten den Rahmen dieses Buches 
völlig übersteigen würde. Diese Listung stellt lediglich einen Anhaltspunkt für mögliche Alternativen dar. Weitere Kapitel über Unterdrucktherapie, Prophylaxe, ungeeignete Wundtherapeutika etc. schließen sich an.

Wir danken allen Mitgliedern der AG „Chronische Wunde“ für die Unterstützung bei der Erstellung. Darüber hinaus danken wir der Fachgruppe Pflege für die Zuarbeit beim Kapitel „Ernährung und Wunde“ sowie Herrn Dr. med. Johann Peter Prinz für die kritische und sehr konstruktive Durchsicht des Manuskriptes. Unser Dank gilt auch dem Thieme Verlag, insbesondere Frau Engeli, für die konstruktive und ansprechende Umsetzung der Idee in Form eines Kittel-Taschenbuches.

Wir wünschen allen Lesern Freude und Gewinn bei der täglichen Arbeit an den Patienten. Möge dieses Buch eine Hilfe dazu sein. Für konstruktive Kritik sind wir sehr dankbar.

Thilo Bode, Thomas Horn, Andrea Schüning 


\section{Inhaltsverzeichnis}

Anforderungen an eine optimale Wundbehandlung bzw. Wundauflage.

1.1 Therapieziele

$1.2 \quad$ Phasen der Wundheilung $\ldots \ldots \ldots \ldots \ldots \ldots \ldots \ldots \ldots \ldots$

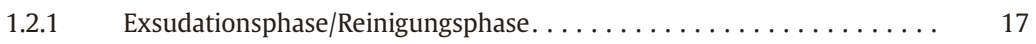

1.2.2 Granulationsphase/Proliferationsphase .................. 19

1.2.3 Epithelisierungsphase ............................ 19

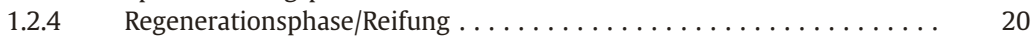

$1.3 \quad$ Einteilung der Wunden $\ldots \ldots \ldots \ldots \ldots \ldots \ldots \ldots \ldots \ldots \ldots \ldots \ldots \ldots \ldots$

$1.4 \quad$ Wundheilungsstörung $\ldots \ldots \ldots \ldots \ldots \ldots \ldots \ldots \ldots \ldots \ldots \ldots \ldots \ldots$

1.5 Wundreinigung: mechanisch - enzymatisch - chirurgisch

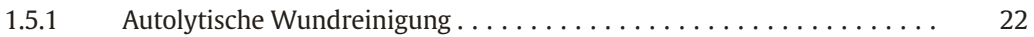

Beschreibung. ............................. 22

Indikation .................................. 23

Anwendungsweise............................ 24

1.5.2 Mechanische Wundreinigung - Entfernen von Belägen mit stumpfen Materialien ................................ 24

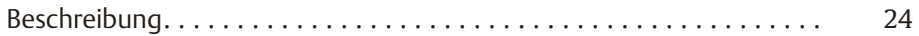

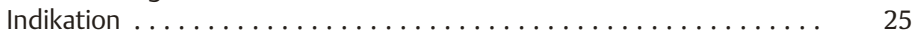

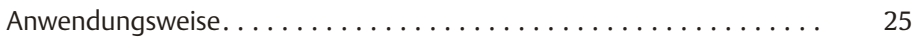

1.5.3 Chirurgische Wundreinigung (mit scharfen Instrumenten) -

Wunddébridement ............................ 25

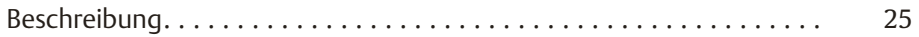

Indikation und Anwendungsweise .................. 26

1.5.4 Ultraschallassistierte Wundreinigung (UAW) - Sonderform des

chirurgischen Wunddébridements. . . . . . . . . . . . . . . . . 27

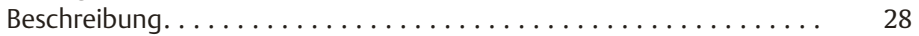

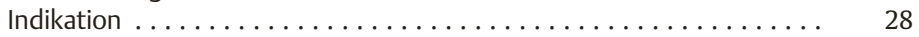

Anwendungsweise........................... 30

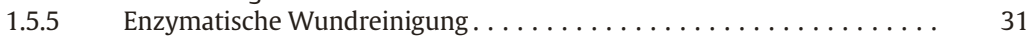

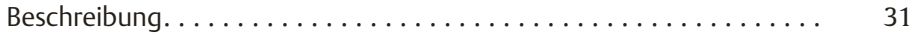

Indikation ................................. 32

Anwendungsweise........................ 32

1.5.6 Biochirurgische Wundreinigung - Fliegenmaden (aus Lucilia sericata). . 33

Beschreibung. ........................... 34

Indikation ................................... 35

Anwendungsweise............................ 35 
1.5.7 Wundspülung. .............................. 36

Wundspülösungen und antiseptische Lösungen ............ 37

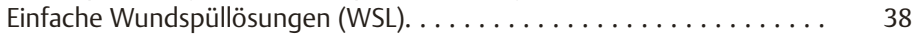

Konservierte und antiseptische Wundspüllösungen . . . . . . . . 40

Antiseptische Gele . . . . . . . . . . . . . . . . . . . . . . . . . 45

1.6 Hygienische Aspekte der Wundversorgung/

Verbandwechsel.......................... 47

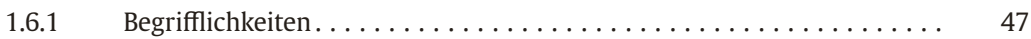

Antiseptik................................ 47

Asepsis................................... 48

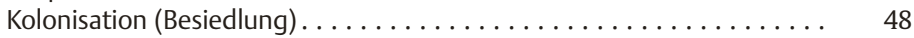

Kontamination ........................... 48

Kritische Kolonisation (Besiedlung). . . . . . . . . . . . . . 48

Wundinfektion ............................. 48

Wundinfektion mit systemischer Allgemeinreaktion. . . . . . . . . 48

Infektionswege/Eintrittsstelle Mikroorganismen/Krankheitserreger ... . 49

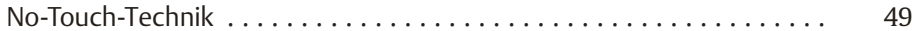

1.6.2 Sachgerechte Entnahme von Material zur mikrobiologischen Untersuchung ...................................... 49

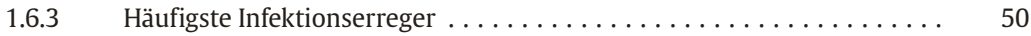

1.6.4 Der Verbandwagen............................. 50

1.6.5 Allgemeine Hinweise zum Verbandwechsel . . . . . . . . . . . . . . 51

1.6.6 Ablauf des Verbandwechsels. . . . . . . . . . . . . . . . . 51

1.7 Wunddokumentation. ........................ 52

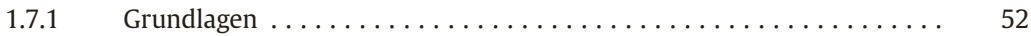

1.7.2 Empfehlung zur Wunddokumentation .................. 53

1.7.3 Fotodokumentation .............................. 54

Einwilligungserklärung ......................... 54

Technische Voraussetzungen . . . . . . . . . . . . . . . . . . . 54

Rechtliche Aspekte. .......................... 54

Häufigkeit der Fotodokumentation. . . . . . . . . . . . . . . . . 55

Praktische Hinweise . . . . . . . . . . . . . . . . . . . . . 55

2 Produkte zur Wundbehandlung............... 57

2.1 Einführung $\ldots \ldots \ldots \ldots \ldots \ldots \ldots \ldots \ldots \ldots \ldots \ldots \ldots \ldots \ldots \ldots \ldots$

2.1.1 Voraussetzungen für eine ideale Wundauflage $\ldots \ldots \ldots \ldots \ldots \ldots .57$

0

1

4

54

5


$2.2 \quad$ Standardverbandmaterial.................... 60

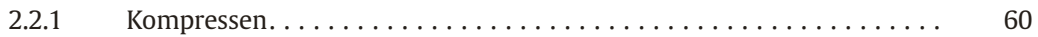

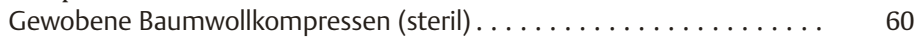

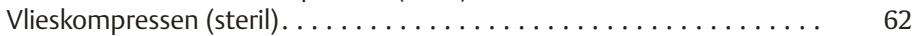

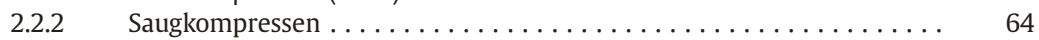

Saugkompressen ohne Superabsorber. . . . . . . . . . . . . . . 64

Saugkompressen mit Superabsorber . . . . . . . . . . . . . . . . . 67

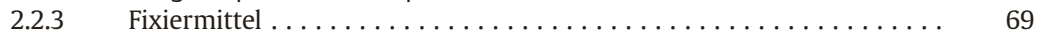

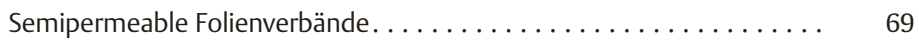

Fixiermittel für normal belastbare Haut . . . . . . . . . . . . . . . . . . . . . . . . . . . . . . . . . .

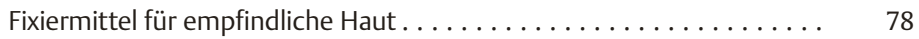

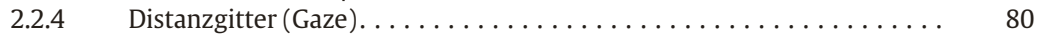

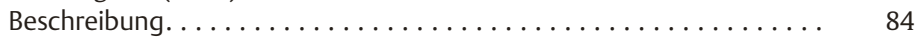

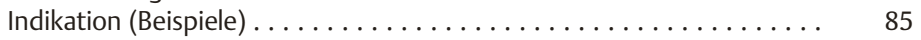

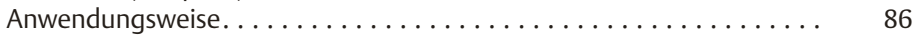

2.3 Alginate $\ldots \ldots \ldots \ldots \ldots \ldots \ldots \ldots \ldots \ldots \ldots \ldots \ldots \ldots \ldots \ldots \ldots \ldots \ldots$

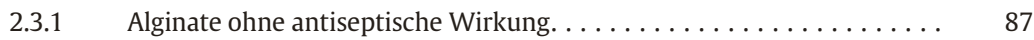

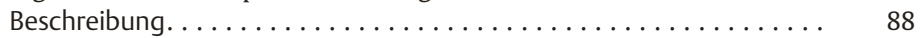

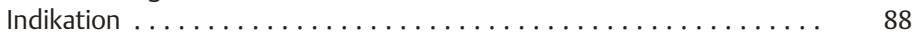

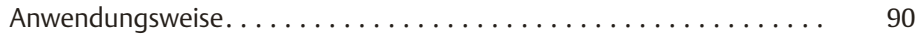

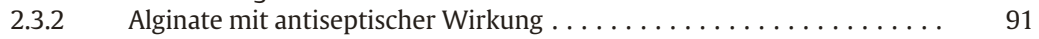

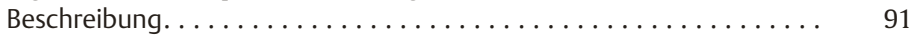

Empfohlene Verwendung . . . . . . . . . . . . . . . 92

$2.4 \quad$ Hydrofaserverbände. . . . . . . . . . . . . . . . 93

2.4.1 Hydrofaser ohne antiseptische Wirkung $\ldots \ldots \ldots \ldots \ldots \ldots \ldots \ldots$

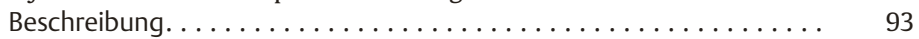

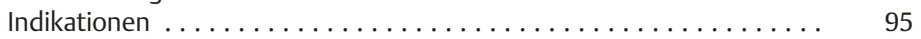

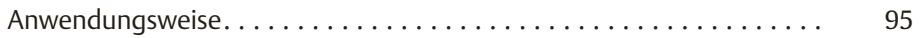

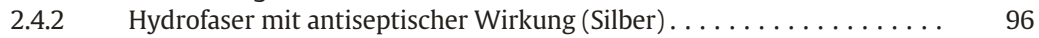

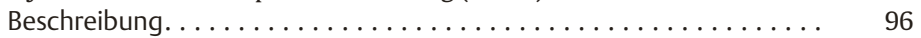

Empfohlene Verwendung . . . . . . . . . . . . . . . . . . 97

2.4.3 Unterschied zwischen Alginat und Hydrofaser . . . . . . . . . . . . 97

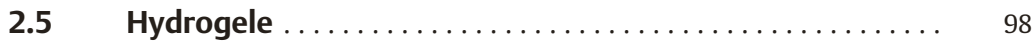

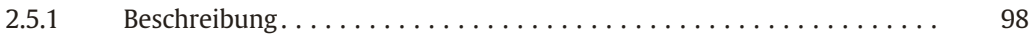

2.5.2 Hydrogele in Tube oder Flasche $\ldots \ldots \ldots \ldots \ldots \ldots \ldots \ldots \ldots \ldots \ldots . \ldots \ldots$

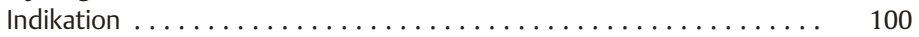

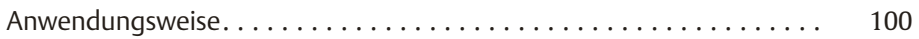

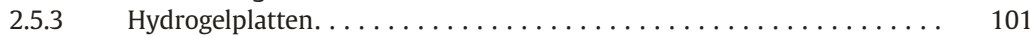

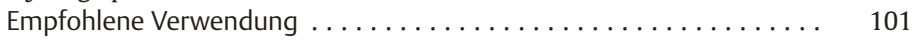

Anwendungshinweise . . . . . . . . . . . . . . . . . 102 
2.6.1 Beschreibung ................................ 103

$2.6 .2 \quad$ Indikation. . . . . . . . . . . . . . . . . . . . . . . . 105

2.6.3 Anwendungsweise . . . . . . . . . . . . . . . . . . . . . 106

2.7 Schaumstoffverbände (Hydropolymere) . . . . . . . . . . . 107

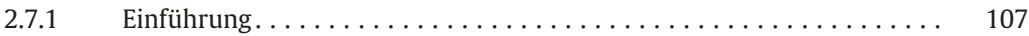

Indikation.................................. 109

Anwendungsweise ............................... 109

2.7.2 Hydropolymerverbände ohne Superabsorber, geschlossenporig . . . . . . 109

Beschreibung ............................... 114

Indikation................................. 114

Anwendungshinweise......................... 116

2.7.3 Hydropolymerverbände mit Superabsorber . . . . . . . . . . . . . . . 117

Empfohlene Verwendung. . . . . . . . . . . . . . . . . . . . . . . . . 117

Beschreibung ................................... 119

Indikation. ................................... 119

Anwendungshinweise. ........................... 119

2.7.4 Offenporige Hydropolymerverbände $\ldots \ldots \ldots \ldots \ldots \ldots \ldots \ldots \ldots . \ldots \ldots$

Beschreibung ........................... 120

Epigard zur Wundkonditionierung vor Transplantation........... 120

Wundbehandlung mit PUR-Schaum (mittel- bis grobporig, gemischt-

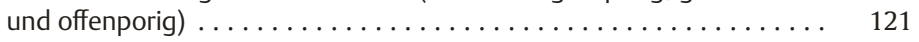

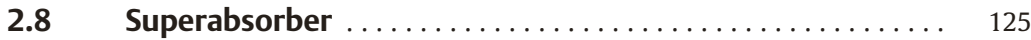

2.8.1 Beschreibung ................................ 127

2.8.2 Anwendungshinweise ......................... 127

2.9 Spezielle Produkte. . . . . . . . . . . . . . . . . . . . 130

2.9.1 Hydrophobe Wundauflagen $\ldots \ldots \ldots \ldots \ldots \ldots \ldots \ldots \ldots \ldots \ldots \ldots$

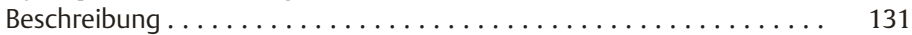

Indikation. ................................ 131

Anwendungsweise .............................. 132

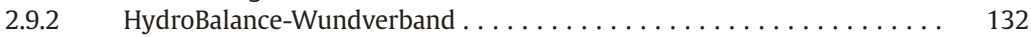

Beschreibung .............................. 133

Indikation.................................. 133

Anwendungsweise . ........................... 135

2.9.3 Hydroaktive Wundauflagen - Laminate - HydroClean . . . . . . . . . 135

Wundkissen mit Saugspülmechanismus ohne antiseptische Wirkung. . . 135

Wundkissen mit Saugspülmechanismus mit antiseptischer Wirkung . . . 137

2.9.4 Kollagen.................................... 138

Kollagene ohne antiseptische Wirkung. . . . . . . . . . . . . 138

Kollagen mit antiseptischer Wirkung $\ldots \ldots \ldots \ldots \ldots \ldots \ldots \ldots .140$ 
2.9.5 Kohleprodukte................................ 140

Einführung. ............................ 140

Kohleprodukte ohne antiseptische Wirkung. . . . . . . . . . . 142

Kohleprodukte mit antiseptischer Wirkung . . . . . . . . . . . . 142

2.9.6 Honigpräparate . . . . . . . . . . . . . . . . . . . . . . . . . . . . 143

Beschreibung. . . . . . . . . . . . . . . . . . . . . . . 143

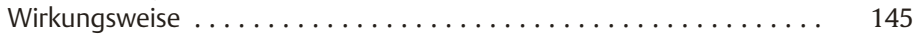

Indikation ................................ 145

Anwendungsweise.......................... 146

Bewertung. .............................. 147

2.9.7 Hautersatzprodukte. ............................ 147

Allgemeine Hautersatzprodukte - Wundkonditionierung vor

Transplantation . . . . . . . . . . . . . . . . . . . . . . . 147

Spezielle Hautersatzprodukte (Verbrennung) . . . . . . . . . . . . 161

3 Unterdruck-Wundtherapie/NPWT.

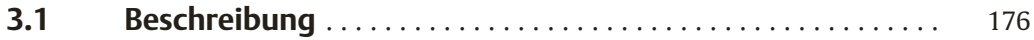

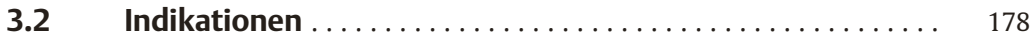

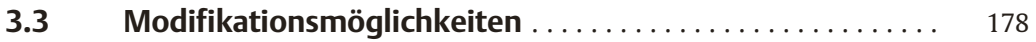

3.3.1 Übersicht................................. 178

3.3.2 Schwamm-Material ............................. 179

3.3.3 Sogmodus ................................... 180

3.3.4 Ableitung mit Softports/Drainagen . . . . . . . . . . . . . . . 181

Indikation ............................... 181

Anlage einer Drainage ........................... 183

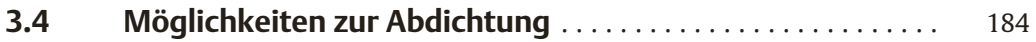

3.4.1 Beschreibung. ................................ 184

3.4.2 Mepiseal ................................. 184

3.4.3 Suprasorb CNP EasyDress - Verband zur Unterdrucktherapie an

Extremitäten . . . . . . . . . . . . . . . . . . . . . . . . . . . . 185

3.4.4 Gelstreifen................................ 186

Eigenschaften . . . . . . . . . . . . . . . . . . . . . . 186

Anwendungshinweis ........................ 186

3.5 Unterdrucktherapie bei Wundinfektion............ 188

3.6 Verbandanlage ................................ 189

3.7 PICO - Kanisterlose Unterdruck-Wundtherapie. 
4.1 Dekubitus.

4.1.1 Definition und klinische Bedeutung . . . . . . . . . . . . . . . 192

4.1.2 Pathogenese................................. 192

4.1.3 Risikoeinschätzung. ............................. 195

4.1.4 Therapie................................... 196

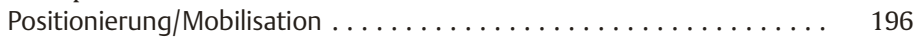

Hilfsmittel . . . . . . . . . . . . . . . . . . . . . . . . 196

Pflege................................... 196

Ernährung ............................... 197

Dekubitusprophylaxe . . . . . . . . . . . . . . . . . . . . . . . 197

4.1.5 Differenzialdiagnosen............................ 197

4.1.6 Internationales Klassifikationssystem für Dekubitus von NPUAP/EPUAP . 197

Kategorie/Stadium I: nicht wegdrückbares Erythem. . . . . . . . . . . . 197

Kategorie/Stadium II: Teilverlust der Haut . . . . . . . . . . . . . . . 199

Kategorie/Stadium III: vollständiger Verlust der Haut . . . . . . . . . 200

Kategorie/Stadium IV: vollständiger Gewebeverlust . . . . . . . . . . 200

Keiner Kategorie/keinem Stadium zuordenbar: Tiefe unbekannt . . . . . 201

Vermutete tiefe Gewebeschädigung: Tiefe unbekannt. . . . . . . . 202

4.2 IAD: Inkontinenz-assoziierte Dermatitis . . . . . . . . . . 202

4.2.1 Pathogenese............................... 202

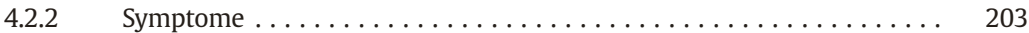

4.2.3 Unterscheidung IAD vs. Dekubitus. . . . . . . . . . . . . . . . . . . . . . 204

4.2.4 Therapie.................................... 204

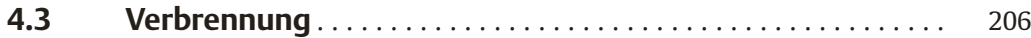

4.3.1 Definition und Pathogenese . . . . . . . . . . . . . . . . . 206

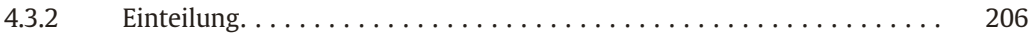

4.3.3 Therapie..................................... 209

Erstmaßnahmen................................. 209

Behandlung in der Klinik. . . . . . . . . . . . . . . . . . . . . . . . . . 209

4.3.4 Verbrennungsprodukte ......................... 211

Prontosan Wound Gel X . . . . . . . . . . . . . . . . . . . . . . . . . 212

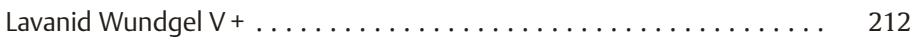

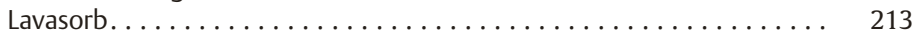

Suprathel und Biobrane .......................... 213 
4.4.1 Konventionelle Versorgung des offenen Abdomens . . . . . . . . . . 217

4.4.2 Intraabdominale Negative Pressure Wound Therapy (NPWT) . . . . . . 218

4.4.3 Kombination der Verfahren Netzeinbau mit Negative Pressure Wound Therapy (NPWT) . . . . . . . . . . . . . . . . . . . . . . . . 219

4.4.4 Komplikation: intestinale Fistel . . . . . . . . . . . . . . . . . . 219

Versorgung mit NPWT und Fisteladapter . . . . . . . . . . . . . . . . . . . 220

Versorgung mit Wunddrainagebeuteln . . . . . . . . . . . . . . . 222

4.5 Wunden bei Tumorpatienten (Palliativsituation). . . . . . 223

4.5.1 Ziele der Therapie . . . . . . . . . . . . . . . . . . 224

4.5.2 Möglichkeiten der Lokalbehandlung wundbezogener Probleme. . . . . . 224

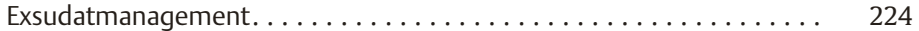

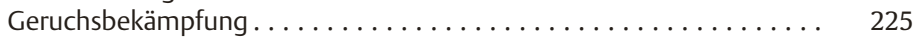

Wundrandschutz............................ 226

Blutungen . . . . . . . . . . . . . . . . . . . . . . . . 226

$5 \quad$ Weitere therapeutische Maßnahmen und

Prophylaxe....

5.1 Hautschutz

5.1.1 Wundumgebung und Störfaktoren der Hautbarriere . . . . . . . . . . . 227

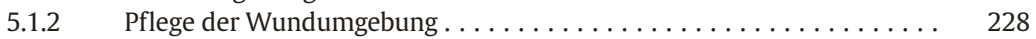

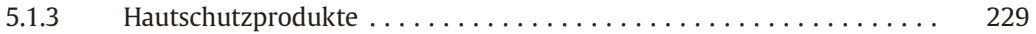

Hautschutz zur Prophylaxe. . . . . . . . . . . . . . . . . . . 229

Hochleistungsfähiger Hautschutz . . . . . . . . . . . . . . . 232

Einsatz von Cavilon Hautschutz vs. Cavilon Advanced . . . . . . . . . . . 235

$5.2 \quad$ Kompressionstherapie .................... 236

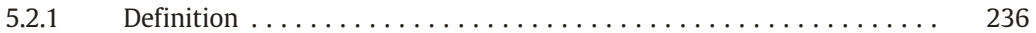

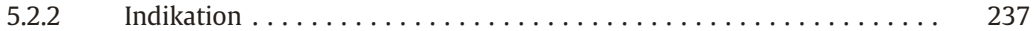

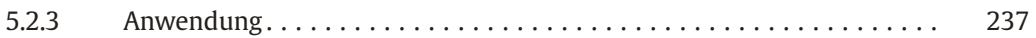

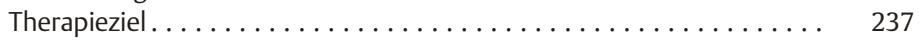

Therapiemöglichkeiten . . . . . . . . . . . . . . . . . . . . . 237

Beschreibung. .............................. 237

Durchführung ........................... 239

5.3 Therapie und Prävention mit Ligasano ............. 243

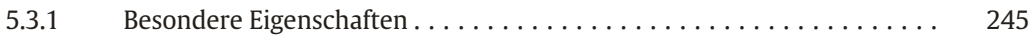

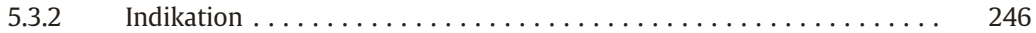

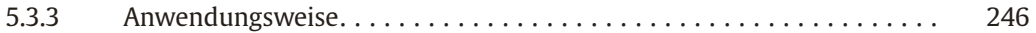


$5.4 \quad$ Ernährung und Wunde.................... 250

5.4.1 Handlungsempfehlung der Helios-Fachgruppe Pflege zum Ernährungsmanagement ............................. 250

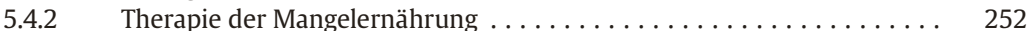

$5.5 \quad$ Edukation von Patienten und Angehörigen . . . . . . . . . . . . 254

$6 \quad$ Ungeeignete Wundtherapeutika............ 255

$6.1 \quad$ Lokalantibiotika . . . . . . . . . . . . . . . . . . . . . 255

$6.2 \quad$ Farbstofflösungen $\ldots \ldots \ldots \ldots \ldots \ldots \ldots \ldots \ldots \ldots \ldots \ldots$

$6.3 \quad$ Veraltete Antiseptika . . . . . . . . . . . . . . . . . . . . 256

$6.4 \quad$ Polyvidon-Jod-Lösung/Jodoformgaze.............. 256

6.5 Weitere ungeeignete Produkte............... 257

6.6 Nicht geeignete Methoden und Vorgehensweisen ....... 257

$7 \quad$ Herstellerverzeichnis ..................... 259

$8 \quad$ Literatur und Adressen ........................ 267

$8.1 \quad$ Literatur . . . . . . . . . . . . . . . . . . . . . . . . . . . . 267

$8.2 \quad$ Nationale Adressen . . . . . . . . . . . . . . . . . . . . . . 267

$8.3 \quad$ Internationale Adressen. . . . . . . . . . . . . . . . . . 268

9 Mitglieder der AG Chronische Wunde ........... 270

Sachverzeichnis ........................... 271

Hinweis: Eine Legende zu den Produkteigenschaften (Piktogramme) befindet sich auf Seite 59. 


\section{Anschriften}

Thilo Bode

Facharzt für Chirurgie,

Zusatzbezeichnung Klinische Akut- und Notfallmedizin

Wundberater AWM ${ }^{\circledR}$

HELIOS Klinikum Pforzheim

Oberarzt der Klinik für Intensiv- und Notfallmedizin

Leitung Wundmanagement

Kanzlerstr. 2-6

75175 Pforzheim

Dr. med. Thomas Horn

HELIOS Klinikum Krefeld

Leiter der AG Chronische Wunde

Leitender Oberarzt der Klinik für Dermatologie und Venerologie

Leitung Wundmanagement

Lutherplatz 40

47805 Krefeld

Andrea Schüning

HELIOS Klinik Leezen

Leitung Wundmanagement

Wittgensteiner Platz 1

19067 Leezen 